\title{
CIRURGIA ABDOMINAL DE ALTA COMPLEXIDADE: CLASSIFICAÇÃO E ESCOLHA DOS EQUIPAMENTOS PELA EQUIPE MULTIPROFISSIONAL
}

\author{
High-complexity abdominal surgery: classification and \\ choice of equipment by the multi-professional team
}

\author{
Cirugía abdominal de alta complejidad: clasificación y \\ elección del equipo quirúrgico por el equipo multiprofesional
}

Adriana Regina Guimarães Martin', Luiz Amaldo Szutan²

RESUMO: Introdução: Os procedimentos de alta complexidade são caracterizados pelo uso da alta tecnologia. A classificação e a escolha dos equipamentos são um desafio para os profissionais envolvidos. Objetivo: Comparar a classificação das cirurgias abdominais de alta complexidade e a escolha dos equipamentos pela equipe multiprofissional entre duas instituições hospitalares. Método: Pesquisa descritiva, quantitativa, transversal e comparativa, com questionários estruturados sobre a opinião dos cirurgiões, anestesiologistas e enfermeiros. Resultado: A classificação dos procedimentos é semelhante quando levados em conta equipamentos $(\mathrm{P}<1,0)$ e estrutura física $(\mathrm{P}=0,172)$ diferenciados. Discussão: A classificação dos procedimentos mais complexos tem concordância entre os profissionais comparado à legislação, mesmo não englobando o porte anestésico, tipo de procedimento e condições clínicas na sua classificação; a escolha dos equipamentos é do profissional que os utiliza, incluindo amplas salas de operações, sendo a cirurgia robótica dispensável aos cirurgiões. Conclusão: Há diferenças entre instituições de saúde em relação à escolha, à disponibilidade de equipamentos e aos tipos de cirurgias abdominais.

Palavras-chave: Centro cirúrgico hospitalar. Organização e administração. Equipamentos cirúrgicos. Procedimentos cirúrgicos operatórios. Gestão de recursos da equipe de assistência à saúde.

ABSTRACT: Introduction: High-complexity procedures are characterized by the use of high-technology. The classification and choice of equipment are challenging for the professionals involved. Objective: To compare the classification of high-complexity abdominal surgeries and the choice of equipment by the multi-professional team between two hospital institutions. Method: Descriptive, quantitative, transversal and comparative research, with structured questionnaires on the opinion of surgeons, anesthesiologists and nurses. Results: The classification of procedures is similar when considering differentiated equipment $(\mathrm{P}<1.0)$ and physical structure $(\mathrm{P}=0.172)$. Discussion: The classification of more complex procedures is in agreement between professionals when compared to the legislation, even if not encompassing anesthetic dimensions, type of procedure or clinical conditions in their classification; the equipment is chosen by the professional who uses them, including operating rooms, with robotic surgeries being expendable to surgeons. Conclusion: There are differences between the choices made by health institutions, the availability of equipment and the types of abdominal surgeries. Keywords: Surgery department, hospital. Organization and administration. Surgical equipment. Surgical procedures, operative. Crew resource management, healthcare.

'Enfermeira pela Universidade de São Paulo (USP); Mestre em Ciências da Saúde pela Faculdade de Ciências Médicas da Santa Casa de São Paulo (FCMSCSP); especialista em Centro Cirúrgico, Recuperação Anestésica e Central de Material e Esterilização pela Universidade de São Paulo (USP). Enfermeira na Irmandade da Santa Casa de Misericórdia de São Paulo - São Paulo (SP), Brasil. E-mail: adrianareginamartin@yahoo.com.br Avenida/Rua Cesário Motta Júnior, 112 - Santa Cecília - CEP: 01221-020 - São Paulo (SP), Brasil.

${ }^{2}$ Mestre em Pesquisa em Cirurgia pela Faculdade de Ciências Médicas da Santa Casa de São Paulo (FCMSCSP). Doutor pela Faculdade de Ciências Médicas da Santa Casa de São Paulo (FCMSCSP). Cirurgião do Aparelho Digestivo. Professor adjunto da Faculdade de Medicina da Irmandade da Santa Casa de Misericórdia de São Paulo - São Paulo (SP), Brasil.

Recebido: 12 maio 2017 - Aprovado: 13 set. 2017

DOI: 10.5327/Z1414-4425201700040004 
RESUMEN: Introducción: procedimientos de alta complejidad se caracterizan por el uso de alta tecnología. La clasificación y elección de los equipos son un desafío para los profesionales. Objetivo: comparar la clasificación de cirugías abdominales de alta complejidad y la elección del equipo por parte del equipo multiprofesional entre dos instituciones hospitalarias. Método: investigación descriptiva, cuantitativa, transversal y comparativa, con cuestionarios estructurados sobre la opinión de cirujanos, anestesiólogos y enfermeras. Resultados: la clasificación de los procedimientos es similar cuando se consideran equipos diferenciados $(\mathrm{P}<1,0)$ y estructura física $(\mathrm{P}=0,172)$. Discusión: la clasificación de procedimientos más complejos está de acuerdo entre los profesionales en comparación con la legislación, incluso si no abarca dimensiones anestésicas, tipo de procedimiento o condiciones clínicas en su clasificación; el equipo es elegido por el profesional que los utiliza, incluidos los quirófanos, y las cirugías robóticas son dispensables para los cirujanos. Conclusión: hay diferencias entre las elecciones realizadas por las instituciones de salud, la disponibilidad de equipos y los tipos de cirugías abdominales. Palabras clave: Servicio de cirugía en hospital. Organización y administración. Equipo quirúrgico. Procedimientos quirúrgicos operativos. Gestión de recursos de personal en salud.

\section{INTRODUÇÃo}

Os procedimentos cirúrgicos são a maior fonte de remuneração de um hospital, seja público ou privado ${ }^{1}$. As cirurgias são classificadas de acordo com o porte em pequeno, médio e grande pelo Ministério da Saúde $(M S)^{2}$ e pela Associação Médica Brasileira $(\mathrm{AMB})^{3}$. Houve uma modificação do MS no Sistema Único de Saúde (SUS) por meio de portarias ministeriais em relação à sua classificação, que passou a ser de pequena, média e alta complexidade (AC) conforme o tipo de cirurgia. O tempo cirúrgico e o potencial de contaminação continuam classificando os procedimentos juntamente com o risco de infecção. Para a AMB, a classificação da cirurgia em pequeno, médio e grande porte permaneceu e foi acrescentada uma tabela de Classificação Brasileira Hierarquizada de Procedimentos Médicos (CBHPM) e Terminologia Unificada de Serviços de Saúde (TUSS) com o porte anestésico de zero a oito, sendo zero com anestesia local em ordem crescente até o oito, que remete a transplantes com anestesia combinada ${ }^{3}$.

A primeira especialidade cirúrgica a ser modificada com a classificação de porte para AC no SUS foi a ortopedia, em 19944, seguida pelas áreas de oncologia ${ }^{5}$, cirurgia cardíaca ${ }^{6}$, neurocirurgia $^{7}$, lesões lábio-palatais ${ }^{7}$, implantes osteointegrados orais ${ }^{7}$ e transplantes ${ }^{7}$. O MS, com o intuito de garantir acesso aos serviços especializados pelo SUS, definiu a AC ambulatorial e hospitalar por meio da Portaria $\mathrm{n}^{\circ} 3.535$, em 02/08/19985: "centros de AC são hospitais que oferecem assistência especializada e integral aos pacientes portadores de determinadas moléstias, atuando na prevenção, detecção precoce, diagnóstico e tratamento do paciente". O modelo de atenção na AC definiu que uma unidade de AC deve apresentar condições técnicas, instalações físicas, equipamentos e recursos humanos adequados à prestação de assistência especializada. O MS define que AC é o conjunto de procedimentos que, no contexto do SUS, envolve alta tecnologia e alto custo com o objetivo de proporcionar à população acesso aos serviços qualificados, integrando-os aos demais níveis de atenção à saúde (básica e de média complexidade).

Os procedimentos estratégicos foram reclassificados como AC para otimização dos mutirões de cirurgias, como as de varizes, hérnias e urológicas ${ }^{8}$. A bariátrica, por exemplo, passou de procedimento estratégico para AC em $2007^{9}$. As cirurgias do aparelho digestivo permaneceram na classificação de média complexidade, com exceção da indicação oncológica, que é de AC apesar de utilizar os mesmos equipamentos.

Com a necessidade de controle e a instituição de normas do setor privado de saúde por parte do MS, foi regulamentada a Lei $n^{\circ} 9.656$, de $03 / 06 / 1998^{10}$, que disciplinou as operadoras de planos de saúde e a organização de novas empresas do segmento. A Agência Nacional de Medicina Suplementar (ANMS), criada em 2001 e subordinada ao MS, estabeleceu a AC para a medicina complementar do sistema privado de saúde e instituiu uma lista de procedimentos para melhorar a remuneração do setor ${ }^{11}$.

A diferença entre a classificação dos procedimentos cirúrgicos entre média e AC com as tabelas de remuneração dos setores público e privado é significativa. Existe grande discrepância de valores repassados às instituições de atendimento ao SUS, o que mostra a baixo financiamento dos serviços públicos e o sucateamento da saúde no Brasil. No setor privado, os procedimentos de grande porte são mais bem remunerados pelas operadoras de saúde, seguindo as tabelas CBHPM e TUSS, havendo concorrência entre as instituições hospitalares para oferecer à população tecnologia de ponta para a escolha das equipes e dos clientes. No setor público, existe grande dificuldade de acesso da população aos serviços mais qualificados de saúde, com a ociosidade na utilização dos equipamentos e recursos existentes. A falta 
de materiais e equipamentos básicos reflete a realidade dos setores da saúde e demonstra o sucateamento da assistência na América Latina ${ }^{12}$.

Uma organização de AC necessita de suporte técnico, estrutura física, material e recursos humanos. Tanto para a alta quanto para a média complexidade é preciso haver estruturas semelhantes, afinal, procedimentos de grande porte pela tabela AMB, como cirurgias abdominais hepáticas, esofágicas, pancreáticas e coloproctológicas, que são de média complexidade no SUS, requerem habilidades e conhecimento específico das equipes envolvidas devido às dificuldades técnicas e anestésica e com tempo cirúrgico prolongado. Para as cirurgias de urgência/emergência, a classificação manteve a média complexidade por exigir equipamentos básicos de uma sala de operação $(\mathrm{SO})^{13}$.

A cirurgia robótica tem sido difundida mundialmente, assim como a cirurgia minimamente invasiva de acesso à população em todas as classes sociais, porém, no Brasil, o custo é muito alto do sistema robótico e limitado a poucas instituições privadas e públicas. O SUS considera as diferenças entre a alta e média complexidade pelo uso de equipamentos de última geração e uma tabela do custo relativo referente ao procedimento. A tecnologia seria o diferencial entre um procedimento de média e alta complexidade.

Um procedimento extremamente simples, mas que utiliza um equipamento recomendável, pode ser considerado de AC. Isso demonstra os equívocos da tabela, como procedimentos onerosos e altamente complexos estarem juntos a outros simples e de baixa complexidade técnica. Com o intuito de garantir a implantação dos serviços de AC e a segurança anestésico-cirúrgica, foram propostos pelo MS os mínimos equipamentos para a segurança do paciente para um centro cirúrgico (CC) de AC nas áreas de oncologia e neurocirurgia no Brasil ${ }^{14}$.

A estrutura organizacional de um CC engloba as equipes cirúrgicas, anestésicas e de enfermagem. Qualquer SO é organizada pela equipe de enfermagem e denominada "sala padrão"14 ou "sala standard", de acordo com os procedimentos de pequeno, médio ou grande portes determinados pela Resolução da Diretoria Colegiada (RDC) $50^{15}$ e pelo Manual de Práticas Recomendadas da Associação Brasileira de Enfermeiros de Centro Cirúrgico, Recuperação Anestésica e Centro de Material e Esterilização (SOBECC). A Agência Americana de Administração em Saúde publicou o padrão standard intermediário e a complexidade cirúrgica para a regulamentação dos procedimentos e o que é necessário para uma sala de cirurgia ${ }^{16}$. Considerando todas as variáveis apresentadas, há dificuldade na caracterização do que é efetivamente necessário em termos de equipamentos para compor uma $\mathrm{SO}$ para procedimentos de $\mathrm{AC}^{17}$ - devese dispor de equipamentos e tecnologia de última geração, com a indicação de indispensáveis, necessários ou recomendáveis para os procedimentos. Apesar da evolução e inovação anuais, de acordo com as práticas recomendadas para a SO, não há necessidade de troca ou aquisição desses equipamentos; a tecnologia deve ser contextualizada a todo o corpo clínico e multiprofissional para que o uso compense o custo. No setor público, não são disponibilizados pela instituição e serem dispensáveis ao procedimento. A oferta de equipamentos no setor privado depende da aquisição e liberação para uso pelas operadoras de saúde devido ao alto custo $^{18,19}$.

\section{OBJETIVO}

O objetivo deste estudo é descrever a definição e classificação das cirurgias abdominais de AC e a escolha dos equipamentos de acordo com a equipe multiprofissional entre duas instituições hospitalares.

\section{MÉTODOS}

A pesquisa é descritiva, quantitativa, transversal e comparativa. Foi realizada em dois CCs: (A) um hospital privado, filantrópico, de porte extra, de assistência quaternária; e em (B) um privado, filantrópico, de grande porte, com seguros privados de saúde. Como critério de inclusão, foram considerados os cirurgiões atuantes na área de Cirurgia Geral e do Sistema Digestivo e Transplantes, enfermeiros e anestesiologistas contratados, atuantes em CC e presentes nos dias de coleta dos dados, com a autorização da Comissão Científica da Instituição, conforme programação do projeto de pesquisa aprovado pelo Comitê de Ética em Pesquisa: protocolo CEP 370/10 da instituição A e CEP 11/10 da instituição B. Como critério de exclusão, levaram-se em conta os profissionais que atuam exclusivamente com cirurgias ambulatoriais e que não devolveram os questionários no prazo de 30 dias.

A coleta de dados foi realizada por meio de um questionário estruturado entregue pessoalmente a cada um dos profissionais. Na instituição A, houve o total de 82 questionários a cirurgiões, 50 a anestesiologistas e 22 a enfermeiros, enquanto na B, 123 a cirurgiões, 108 da anestesia e 26 a enfermeiros. A amostragem foi simples e aleatória, composta pelos que 
devolveram os questionários, totalizando: 25 cirurgiões na $\mathrm{A}$ e mesma quantidade na B, sendo 13 cirurgiões gerais e/ou equipe do aparelho digestivo e 12 transplantadores; 25 anestesiologistas na A e 21 na B; e 22 (A) e 24 (B) enfermeiros.

A validação do conteúdo dos instrumentos de pesquisa e da forma da primeira para a segunda versão do questionário foi realizada com o auxílio de especialistas da área de CC por meio da técnica de Delphi. Pela semelhança das perguntas e respostas abertas foram agrupadas em questões fechadas. O pré-teste foi um estudo descritivo e não aplicado teste estatístico na primeira fase com especialistas, que contemplava dados da instituição, identificação, conceito e classificação de $\mathrm{AC}$, equipamentos, formação da equipe, recursos humanos e mobiliários de acordo com a prática clínica, literatura e legislação. Inicialmente, as questões eram abertas de forma descritiva, sendo alteradas para um questionário estruturado com questões fechadas na segunda fase com a abrangência de cinco categorias: definição da AC (9 questões), equipamentos necessários e/ ou recomendáveis aos procedimentos citados e/ou utilizados pelas diversas equipes multiprofissionais (33 itens), planta física da SO (2 questões), formação acadêmica (1 questão) e tipos de procedimentos cirúrgicos (15 itens). Para os enfermeiros, foram incluídas duas categorias: montagem de SO com mobiliário (10 itens) e o papel do enfermeiro em CC (6 itens e 7 questões). Os questionários estruturados para os enfermeiros continham 82 itens e 12 perguntas; para os cirurgiões, 60 perguntas; e para os anestesiologistas, 12 perguntas e 59 itens. O período de coleta de dados teve início em 2012, após a aprovação do CEP, nas instituições A e B, sendo entregues 417 questionários e devolvidos 142, respondidos; e devolução de 50 formulários em branco, sendo a maior parte da instituição B. Os questionários foram tabulados em banco de dados, planilha Excel, e separados nas seis categorias: cirurgiões, anestesiologistas e enfermeiros das instituições A e B. Aplicou-se o Teste do $\chi^{2}$ de Pearson, quando necessário, e o teste exato de Fischer, independente da categoria, adotando-se em todos nível de significância de $5 \%$.

\section{RESULTADOS}

Foram entregues 417 questionários e devolvidos 142 (34\%), respondidos e tabulados em banco de dados Excel separados nas três categorias para cada uma das instituições: cirurgiões, anestesiologistas e enfermeiros. Os perfis de cirurgiões foram agrupados de acordo com a procedência ( $50 \%$ da instituição $\mathrm{A}$ e $50 \%$ da B); idade (entre 30 e 40 anos na instituição A e acima de 40 anos na instituição B); área de atuação - com prevalência em cirurgia do aparelho digestivo na instituição A (36\%) e transplantadores na instituição B; tempo e área de atuação acima de 10 anos em ambas as instituições. Da mesma forma, os anestesiologistas foram agrupados de acordo com a procedência ( $54,3 \%$ da instituição A e 45,7\% da B); idade (entre 31 e 40 anos em ambas as instituições); especialidade em anestesia geral, sendo $84 \%$ na instituição A e $81 \%$ na B; tempo de atuação na anestesia - acima de 6 anos na instituição A (72\%) e entre 6 a 10 anos na B (66\%). Por último, os enfermeiros registraram os seguintes números: $22(47,8 \%)$ procedentes da instituição A e $24(52,2 \%)$ da B; idade acima de 31 anos $(72,7 \%)$ na $A$ e $21(87,5 \%)$ na $B$, sendo a maior porcentagem de menores de 30 anos na instituição A, totalizando 6 (27\%); especialização em CC, somando $54,5 \%$ na A e $95,8 \%$ na B; tempo de formação acadêmica de até 5 anos (36,3\%) e acima de 10 anos (45,5\%) na instituição $A$, diferente da B, em que $60 \%$ dos enfermeiros têm de 6 a 10 anos, $17,4 \%$ até 5 anos; e $21,8 \%$ acima de 10 anos.

As Tabelas 1 a 3 descrevem, respectivamente, o conceito e a classificação dos procedimentos de AC, escolha dos equipamentos cirúrgicos e respostas não coincidentes quanto à cirurgia robótica, determinação de SO padrão para AC, escolha de equipamentos recomendáveis e planta física.

Os cirurgiões, anestesiologistas e enfermeiros concordam com a divisão das cirurgias em AC e uma subdivisão e listagem dos procedimentos abdominais. Foram considerados como AC somente as cirurgias específicas com grandes ressecções, como as cirurgias hepáticas, esofágicas, coloproctológicas, pancreáticas, gástricas e transplantes. A listagem é importante para a determinação dos procedimentos pela dificuldade técnica baseada na experiência dos profissionais. Quanto ao tempo cirúrgico, uso de tecnologia de alto custo e porte anestésico, não foram determinantes na classificação dos procedimentos de $\mathrm{AC}$ ao contrário das condições clínicas.

A bomba propulsora para a circulação extracorpórea, a ultrafiltração dialítica e a radiofrequência são equipamentos pouco utilizáveis para os procedimentos abdominais. O sistema de reaproveitamento sanguíneo (cell saver) é utilizado por apenas $21 \%$ dos cirurgiões da instituição B, diferentemente da instituição A, por ser indisponível. Apenas 16\% dos cirurgiões da instituição B indicaram indisponível por falta de pessoal para a utilização, enquanto $30 \%$ relatam que não precisam, $21 \%$ a utilizam e 49\% pode ser dispensável e não utilizam.

Quanto à suficiência do espaço físico, a planta foi considerada satisfatória por $100 \%$ dos profissionais na instituição A 
e $80 \%$ na B, mas a SO deveria ser mais ampla. Para os enfermeiros, verificou-se coincidência nas respostas em relação à organização e gestão do setor: todos opinaram favoravelmente sobre a compra de equipamentos; elaboração de protocolos operacionais de assistência e segurança; organização do mapa de cirurgias; participação na disposição da estrutura desde a planta física, na implementação de um CC com o devido preparo da $\mathrm{SO}$ com equipamentos básicos padrão, não aguardando a equipe médica na escolha de $\mathrm{SO}$ e sua utilização para os diversos tipos de procedimentos. Os anestesiologistas não

Tabela 1. Conceito e classificação dos procedimentos de alta complexidade de acordo com a equipe multiprofissional — São Paulo, 2012.

\begin{tabular}{|l|c|c|c|c|c|c|c|}
\hline Classificação & Cirurgiões A & Cirurgiōes B & Anestesistas A & Anestesistas B & Enfermeiros A & Enfermeiros B & Valor p \\
\hline $\begin{array}{l}\text { Conceito } \\
\text { média e alta } \\
\text { complexidade }\end{array}$ & $\begin{array}{c}\text { Concordância } \\
96 \%\end{array}$ & $\begin{array}{c}\text { Concordância } \\
88 \%\end{array}$ & $\begin{array}{c}\text { Concordância } \\
68 \%\end{array}$ & $\begin{array}{c}\text { Concordância } \\
90 \%\end{array}$ & $\begin{array}{c}\text { Concordância } \\
100 \%\end{array}$ & $\begin{array}{c}\text { Concordância } \\
72 \%\end{array}$ & $>0,05$ \\
\hline $\begin{array}{l}\text { Divisão, } \\
\text { subdivisão e } \\
\text { listagem }\end{array}$ & $94 \%$ & $94 \%$ & $94 \%$ & $94 \%$ & $94 \%$ & $81 \%$ & $>0,05$ \\
\hline $\begin{array}{l}\text { Condição } \\
\text { clínica }\end{array}$ & $71 \%$ & $85 \%$ & $80 \%$ & $45 \%$ & $85 \%$ & $100 \%$ & $>0,05$ \\
\hline $\begin{array}{l}\text { Porte } \\
\text { anestésico }\end{array}$ & $67 \%$ & $92 \%$ & $76 \%$ & $60 \%$ & $81 \%$ & $90 \%$ & $>0,05$ \\
\hline $\begin{array}{l}\text { Tempo } \\
\text { cirúrgico }\end{array}$ & $62 \%$ & $83 \%$ & $76 \%$ & $40 \%$ & $76 \%$ & $80 \%$ & $>0,05$ \\
\hline $\begin{array}{l}\text { Tecnologia } \\
\text { alto custo }\end{array}$ & $55 \%$ & $30 \%$ & $60 \%$ & $70 \%$ & $38 \%$ & $30 \%$ & $>0,05$ \\
\hline
\end{tabular}

Tabela 2. Escolha de equipamentos utilizados para cirurgia de alta complexidade de acordo com a equipe multiprofissional — São Paulo, 2012.

\begin{tabular}{|c|c|c|c|c|c|c|c|}
\hline Equipamento & Cirurgiōes A & Cirurgiōes B & Anestesistas A & Anestesistas B & Enfermeiros A & Enfermeiros B & Valor $\mathbf{p}$ \\
\hline Videolaparoscopia & $92 \%$ & $92 \%$ & Não utilizam & Não utilizam & $100 \%$ & $92 \%$ & $>0,05$ \\
\hline Microscópio & $\begin{array}{c}75 \% \\
\text { dispensável }\end{array}$ & $\begin{array}{c}73 \% \\
\text { dispensável }\end{array}$ & $\begin{array}{c}64 \% \\
\text { dispensável }\end{array}$ & $\begin{array}{c}64 \% \\
\text { dispensável }\end{array}$ & $\begin{array}{c}100 \% \\
\text { indispensável }\end{array}$ & $\begin{array}{c}91 \% \\
\text { indispensável }\end{array}$ & $>0,05$ \\
\hline $\begin{array}{l}\text { Bisturi mono/ } \\
\text { bipolar }\end{array}$ & $90 \%$ & $90 \%$ & $\begin{array}{c}64 \% \text { não } \\
\text { é de alta } \\
\text { complexidade }\end{array}$ & $\begin{array}{l}57 \% \text { não } \\
\text { é de alta } \\
\text { complexidade }\end{array}$ & $100 \%$ & $100 \%$ & $=0,488$ \\
\hline $\begin{array}{l}\text { Bisturi } \\
\text { ultrassônico }\end{array}$ & $80 \%$ & Indisponível & Indiferente & Indiferente & Indisponível & $70 \%$ & $=0,012$ \\
\hline $\begin{array}{l}\text { Bisturi jato de } \\
\text { água }\end{array}$ & $60 \%$ & $20 \%$ & $60 \%$ & Indiferente & $80 \%$ & $70 \%$ & $=0,03$ \\
\hline $\begin{array}{l}\text { Ultrafiltração } \\
\text { dialítica }\end{array}$ & $16,4 \%$ & $12,5 \%$ & $36 \%$ & $36 \%$ & $4,8 \%$ & $33,3 \%$ & $>0,05$ \\
\hline Eletroestimulador & $41,2 \%$ & $41,2 \%$ & $100 \%$ & $100 \%$ & $95,2 \%$ & $83,3 \%$ & $>0,05$ \\
\hline Biopump* & $41 \%$ & $12 \%$ & $36 \%$ & $36 \%$ & $100 \%$ & $85 \%$ & $>0,05$ \\
\hline Radiofrequência & $52 \%$ & $37 \%$ & $36 \%$ & $36 \%$ & $71 \%$ & $83 \%$ & $>0,05$ \\
\hline $\begin{array}{l}\text { Escopia e } \\
\text { intensificador de } \\
\text { imagem }\end{array}$ & $79,2 \%$ & $73,9 \%$ & $36 \%$ & $36 \%$ & $100 \%$ & $91,6 \%$ & $>0,05$ \\
\hline Cell saver** & Indisponível & $21 \%$ & Indisponível & $100 \%$ & Indisponível & $100 \%$ & $>0,05$ \\
\hline
\end{tabular}

*Bomba propulsora para circulação extracorpórea; **sistema de reaproveitamento sanguíneo. 
determinam a SO que será utilizada, e sim os enfermeiros e cirurgiões. A cirurgia robótica foi considerada dispensável para $70 \%$ dos cirurgiões e enfermeiros da instituição A e $60 \%$ da B, diferentemente dos enfermeiros da instituição B, na qual $77 \%$ consideram a robótica necessária, disponível e de uso restrito devido à dificuldade técnica e falta de cobertura pelos seguros privados de saúde.

\section{DISCUSSÃO}

Os procedimentos de média e AC são vistos diferentemente de uma unidade com atendimento SUS para sistema suplementar de saúde. Apesar de a coleta de dados ter sido realizada há mais de cinco anos, o tema continua sendo uma preocupação devido à tabela SUS não englobar procedimentos complexos compatíveis com a remuneração do sistema público, o que é, de fato, necessário para que uma sala de cirurgia garanta a segurança anestésico-cirúrgica com recursos materiais e humanos e seja considerada suficiente aos procedimentos ${ }^{14,16,17}$.

A estrutura da sala de cirurgia "standard" padrão faz parte do manual de práticas recomendadas da SOBECC ${ }^{17}$, com descrição de mobiliário e equipamentos mínimos, e reflete que nem toda tecnologia disponível é necessária. No caso dos seguros privados de saúde, há procedimentos e equipamentos não englobados para a cirurgia e que dependem das autorizações das seguradoras do setor. A comparação entre as instituições filantrópicas fez com que a resposta à questão sobre qual é a

Tabela 3. Respostas não coincidentes quanto à cirurgia robótica, determinação de sala de operação padrão para alta complexidade, escolha de equipamentos recomendáveis, planta física e cirurgia de urgência/emergência para cirurgiões, anestesiologistas e enfermeiros - São Paulo, 2012.

\begin{tabular}{|c|c|c|c|c|c|c|c|c|c|c|c|c|c|c|c|c|}
\hline \multirow{3}{*}{$\begin{array}{l}\text { Procedimentos de } \\
\text { alta complexidade }\end{array}$} & \multirow{3}{*}{$\frac{\text { 은 }}{\mathrm{g}}$} & \multicolumn{5}{|c|}{ Cirurgia } & \multicolumn{5}{|c|}{ Anestesia } & \multicolumn{5}{|c|}{ Enfermagem } \\
\hline & & \multicolumn{2}{|c|}{$\mathbf{A}$} & \multicolumn{2}{|c|}{$\mathbf{B}$} & \multirow[b]{2}{*}{ Valor p } & \multicolumn{2}{|c|}{$\mathbf{A}$} & \multicolumn{2}{|c|}{$\mathbf{B}$} & \multirow[b]{2}{*}{ Valor p } & \multicolumn{2}{|c|}{$\mathbf{A}$} & \multicolumn{2}{|c|}{ B } & \multirow[b]{2}{*}{ Valor p } \\
\hline & & 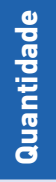 & $a^{e}$ & 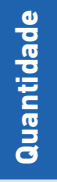 & $a^{\circ}$ & & 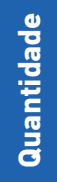 & 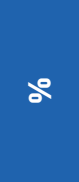 & $\begin{array}{l}\frac{9}{0} \\
\frac{\pi}{0} \\
\text { हैँ } \\
\text { है }\end{array}$ & 2 & & 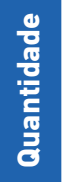 & $a^{\circ}$ & 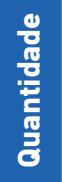 & a & \\
\hline \multirow{2}{*}{$\begin{array}{l}\text { Considera necessária a } \\
\text { cirurgia robótica? }\end{array}$} & Sim & 8 & 32,0 & 11 & 39,1 & \multirow{2}{*}{$0,679^{*}$} & \multirow[b]{2}{*}{ NA } & & \multirow[b]{2}{*}{ NA } & & & 5 & 22,7 & 15 & 62,5 & \multirow{2}{*}{$0,017^{*}$} \\
\hline & Não & 17 & 68,0 & 14 & 60,9 & & & & & & & 17 & 77,3 & 9 & 37,5 & \\
\hline Você costuma & Sim & 12 & 48,0 & & 28,0 & & & & & & & 20 & 90,9 & 22 & 91,6 & \multirow[b]{2}{*}{$<1,0^{* *}$} \\
\hline $\begin{array}{l}\text { operação para realizar } \\
\text { o procedimento de alta } \\
\text { complexidade? }\end{array}$ & Não & 13 & 52,0 & 18 & 72,0 & $0,172^{*}$ & NA & NA & NA & & & 2 & 9,1 & 2 & 8,4 & \\
\hline \multirow{2}{*}{$\begin{array}{l}\text { Escolha dos } \\
\text { equipamentos } \\
\text { recomendáveis ocorre } \\
\text { ao seu critério? }\end{array}$} & Sim & 21 & 84,0 & 25 & 100 & \multirow[b]{2}{*}{$<1,0^{* *}$} & 23 & 92,0 & 21 & 100 & \multirow[b]{2}{*}{$<1,0^{* *}$} & & & & & \\
\hline & Não & 4 & 16,0 & 0 & & & 2 & 8,0 & & & & & NA & NA & & \\
\hline \multirow{2}{*}{$\begin{array}{l}\text { Uma sala de operação } \\
\text { padrão é preparada } \\
\text { com materiais e } \\
\text { equipamentos básicos? }\end{array}$} & Sim & 21 & 84,0 & 24 & 96,0 & \multirow[b]{2}{*}{$0,348^{*}$} & 18 & 72,0 & 18 & 85 & \multirow[b]{2}{*}{$0,303^{*}$} & 19 & 81,0 & 22 & 91,6 & \multirow[b]{2}{*}{$<1,0^{* *}$} \\
\hline & Não & 4 & 16,0 & 1 & 4,0 & & 7 & 28,0 & 3 & 15 & & 3 & 19,0 & 2 & 8,4 & \\
\hline \multirow{2}{*}{$\begin{array}{l}\text { Considera a planta } \\
\text { física do setor de centro } \\
\text { cirúrgico suficiente } \\
\text { às necessidades dos } \\
\text { serviços prestados? }\end{array}$} & Sim & 19 & 76,0 & 23 & 92,0 & \multirow[b]{2}{*}{$0,245^{*}$} & 21 & 88,0 & 17 & 81,0 & \multirow[b]{2}{*}{$0,513^{*}$} & 19 & 81,0 & 22 & 91,6 & \multirow[b]{2}{*}{$0,067^{*}$} \\
\hline & Não & 6 & 24,0 & 2 & 8,0 & & 4 & 12,0 & 4 & 19,0 & & 3 & 19,0 & 2 & 8,4 & \\
\hline \multirow{2}{*}{$\begin{array}{l}\text { Cirurgias de urgência/ } \\
\text { emergência são de alta } \\
\text { complexidade? }\end{array}$} & Sim & 16 & 64,0 & 12 & 48,0 & \multirow{2}{*}{$0,312^{*}$} & 10 & 40,0 & 10 & 47,6 & & 12 & 54,5 & 17 & 70,8 & \\
\hline & Não & 9 & 36,0 & 13 & 52,0 & & 15 & 60,0 & 11 & 52,4 & $0,736^{*}$ & 10 & 45,5 & 7 & 29,2 & \\
\hline
\end{tabular}


diferença entre as cirurgias de um serviço público e privado fosse descrita. Existem distinções entre quantidade e tipos de equipamentos, mobiliários e disponibilidade de tecnologia de ponta. No sistema privado, temos a cirurgia robótica, equipamento de reaproveitamento sanguíneo e sistema de monitorização cerebral, não utilizados para todos os procedimentos, porém disponíveis. Já para o sistema público, alguns equipamentos são disponíveis, mas não em quantidade suficiente para todas as salas de cirurgia. Os cirurgiões da instituição B consideraram o cell saver (sistema de reaproveitamento sanguíneo) pouco utilizado para os procedimentos abdominais de AC; e ele está disponível conforme a preferência do cirurgião $0^{20}$.

A cirurgia robótica vem evoluindo enquanto os profissionais aperfeiçoam a sua técnica, sendo disponível a algumas instituições privadas e públicas de saúde, que a utilizam de forma experimental e acadêmica. As salas híbridas e os tipos de cirurgias evoluem de acordo com a tecnologia disponível no mercado ${ }^{18,19}$.

A pergunta sobre a escolha dos equipamentos recomendáveis ocorre ao critério do profissional seria que nem sempre isso acontece. O sistema de financiamento das instituições é determinante para a cobertura e disponibilidade dos equipamentos $^{13}$. A instituição A, por ser de ensino, permite maior decisão quanto ao equipamento utilizado pela disponibilidade, não dependendo da liberação para uso pelos seguros privados de saúde. Para os anestesiologistas da instituição B, na qual estão disponíveis todos os equipamentos necessários para a segurança anestésica, não haver diferença no critério anestesiológico para o das condições clínicas e o tempo cirúrgico ser menor, a segurança do procedimento é maior. A tecnologia inova, mas nem sempre é necessária, apesar de ela poder auxiliar os profissionais em uma melhor prestação de assistência. Os anestesiologistas não opinam sobre os equipamentos utilizados pelo cirurgião, que, por sua vez, não comentam sobre o tipo de monitorização; cada um faz a sua parte. Os enfermeiros têm autonomia na decisão da SO juntamente com toda a equipe multiprofissional.

$\mathrm{Na}$ instituição A, as salas de cirurgia são dimensionadas para os procedimentos de média complexidade e insuficientes para cirurgias complexas que requerem outros equipamentos. A preocupação com a limitação de recursos humanos e a necessidade de redimensionamento baseada no porte e na complexidade dos procedimentos ocorre em outros paí$\operatorname{ses}^{16-19}$. A classificação deveria incluir o porte anestésico e as condições clínicas, assim como as Diretrizes da Administração do Departamento de Saúde Americano de Washington EUA $^{16}$, publicadas em 2010 e que definiram os padrões de porte cirúrgico.
Uma SO "standard" ou padrão é preparada com materiais e equipamentos recomendáveis e agregados a outras tecnologias de acordo com a necessidade de uso e a sua disponibilidade ao procedimento conforme o manual das Práticas Recomendadas da SOBECC ${ }^{17}$ e de novas tecnologias para as $\mathrm{SOs}^{18}$. Deve seguir as normas para os estabelecimentos de saúde conforme a RDC n ${ }^{\circ} 50$, de $05 / 02 / 2002^{15}$, determinando SOs amplas para grande porte - mesmo 15 anos após a regulamentação, não houve essas alterações. As limitações do estudo são restritas às instituições nas quais os profissionais atuam, mas refletem a realidade da dificuldade de acesso à tecnologia e/ ou disponibilidade sem o uso de equipamentos de alto custo. A resistência da aprovação em outros Comitês de Ética em Pesquisa de instituições privadas, nas quais a comparação pode gerar conflitos de interesses administrativos e relacionar com o serviço público de saúde, pode ter resultados negativos. $\mathrm{O}$ trabalho deve ser expandido para as instituições filantrópicas, públicas, privadas e com seguros de saúde privados, nos quais a limitação e a indisponibilidade dos equipamentos sejam maiores.

\section{CONCLUSÃO}

Os dados definem que há diferenças entre as salas de cirurgias de instituições públicas e privadas no Brasil. A definição e classificação dos procedimentos de AC são importantes para estruturar o ambiente cirúrgico de 1994 a 2012, conforme a legislação. O conceito AC foi considerado incompleto pelos profissionais por não englobar o porte anestésico, o tipo de procedimento e as condições clínicas. O tempo cirúrgico é relevante para a classificação dos procedimentos; os equipamentos são determinados pelos profissionais que os utilizam e devem ser disponibilizados pela instituição; e as cirurgias de urgência e emergência não foram consideradas de $\mathrm{AC}$ por utilizar equipamentos básicos como na média complexidade e não utilizar tecnologia de ponta onde a falta e/ ou escassez de materiais é mais evidente.

Diante da opção de uma SO padrão com equipamentos básicos e espaço físico diminuído, as cirurgias de urgência / emergência podem ser realizadas em SO não projetada para a AC por não ser programada, com maior risco anestésico, e dispensar o uso de equipamentos recomendáveis utilizados nos procedimentos eletivos. A complementação da formação nas especialidades de centro cirúrgico se torna imprescindível quando os procedimentos são de AC. A cirurgia robótica é uma tecnologia que vem evoluindo, embora ainda seja considerada dispensável aos médicos. 


\section{REFERÊNCIAS}

1. Dube M. Global implications from the US hospitals privatization experience. World Hosp Health Serv [Internet]. 2012 [cited on 2017 Aug 22];48(2):08-10. Available from: https://www.ncbi.nlm.nih.gov/ pubmed/22913123

2. Associação Médica Brasileira. Classificação Brasileira Hierarquizada de Procedimentos Médicos. 2014 [cited on 2016 Sept 11]. Available from: http://amb.org.br/_arquivos/_downloads/CBHPM-2014.pdf

3. Associação Médica Brasileira. Lista de Procedimentos Médicos. Valores referenciais [internet]. 1999 [cited on 2017 Aug 11]. Available from: https://amb.org.br/cbhpm/

4. Brasil. Ministério da Saúde. Portaria n 221, de 15 de fevereiro de 2005. Institui a Política Nacional de Atenção de Alta Complexidade em Traumato-Ortopedia e dá outras providências. Diário Oficial da União [Internet]. 2005 [cited on 2017 Jun 11]. Available from: http://bvsms.saude.gov.br/bvs/saudelegis/gm/2005/ prt0221_15_02_2005.html

5. Brasil. Ministério da Saúde. Secretaria de Estado da Saúde. Portaria GM/MS nº 3.535 de 02 de setembro de 1998. Diário Oficial da União [Internet]. 1998 [cited on 2017 Jun 01]. Available from: http://www. saude.mg.gov.br/images/documentos/portaria_3535.pdf

6. Brasil. Ministério da Saúde. Portaria no 1.169, de 15 de junho de 2004. Institui a Política Nacional de Atenção Cardiovascular de Alta Complexidade, e dá outras providências. [Diário Oficial da União [Internet]. 2004 [cited on 2016 Jun 18]. Available from: http://bvsms. saude.gov.br/bvs/saudelegis/gm/2004/prt1169_15_06_2004.html

7. Brasil. Conselho Nacional de Secretários de Saúde. A gestão do SUS: para entender a Gestão do SUS [Internet]. Brasília: CONASS, 2015 [cited on 2017 May 1]. Available from: http://www.conass.org.br/ biblioteca/pdf/A-GESTAO-DO-SUS.pdf

8. Brasil. Ministério da Saúde. Portaria n 252, de 6 de fevereiro de 2006 Institui a Política Nacional de Procedimentos Cirúrgicos Eletivos de Média Complexidade Ambulatorial e Hospitalar. Diário Oficial da União [Internet]. 2006 [cited on 2016 Nov 15]. Available from: http:// dtr2006.saude.gov.br/SAS/Portaria252/anexoll-PT096.html

9. Brasil. Ministério da Saúde. Portaria n 1.570 de 28 de junho de 2007. Determina que a Secretaria de Atenção à Saúde, isoladamente ou em conjunto com outras Secretarias do Ministério da Saúde, adote todas as providências necessárias à organização da assistência ao portador de obesidade grave. Diário Oficial da União [Internet]. 2007 [cited on 2016 Nov 1]. Available from: http://www1.saude.rs.gov.br/ dados/1190226882504GM-1570.html

10. Brasil. Lei n 9.656, de 3 de junho de 1998. Dispõe sobre os planos e seguros privados de assistência à saúde. Diário Oficial da União [Internet]. 1998 [cited on 2016 Oct 30]. Available from: http://www2. camara.leg.br/legin/fed/lei/1998/lei-9656-3-junho-1998-353439publicacaooriginal-1-pl.html
11. Brasil. Ministério da Saúde. Resolução Normativa n 338 , de 21 de outubro de 2013. Diário Oficial da União [Internet]. 2013 [cited on 2016 Nov 15]. Available from: http://www.lex.com.br/legis_24984445_RESOLUCAO_ NORMATIVA_N_338_DE_21_DE_OUTUBRO_DE_2013.aspx

12. Betancur JD, Montaño LM, Jaramillo AF, Delgado CE. Fast track surgery, a strategy to improve operational efficiency in a high-complexity hospital in Latin America. World Hosp Health Serv. 2015;51(1):40-3.

13. Silva JFSS, Mattos IE. Avaliação da assistência oncológica de alta complexidade em um município de fronteira em Mato Grosso do Sul: uma proposta de cálculo de estimativas de cobertura. Cad Saúde Coletiva. 2012 [cited on 2017 Aug 23];20(3):314-20 Available from: http://www.cadernos.iesc.ufrj.br/cadernos/images/csc/2012_3/ artigos/csc_v20n3_314-320.pdf

14. Brasil. Ministério da Saúde. Gabinete do Ministro. Portaria n 2.947, de 21 de dezembro de 2012. Atualiza, por exclusão, inclusão e alteração, procedimentos cirúrgicos oncológicos na tabela de procedimentos, medicamentos, órteses/próteses e materiais especiais do SUS. Diário Oficial da União [Internet]. 2012 [cited on 2016 Nov 15]. Available from: http://bvsms.saude.gov.br/bvs/saudelegis/gm/2012/ prt2947_21_12_2012.html

15. Brasil. Ministério da Saúde. Agência Nacional de Vigilância Sanitária. RDC 50, de 21 de fevereiro de 2002. Dispõe sobre o regulamento técnico para planejamento, programação, elaboração e avaliação de projetos físicos de estabelecimentos assistenciais de saúde [Internet]. 2002 [cited on 2016 Nov 18]. Available from: http://www. redeblh.fiocruz.br/media/50_02rdc.pdf

16. U.S.A. Department of Veterans Affairs. Veteran Health Administration. Facility infrastructure requirements to perform standard, intermediate, or complex surgical procedures [Internet]. 2010 [cited on 2017 Apr 18]. Available from: https://www.va.gov/vhapublications/ViewPublication. asp?pub_ID=22

17. Sociedade Brasileira de Enfermeiros de Centro Cirúrgico, Recuperação Anestésica e Central de Material. Manual de Práticas Recomendadas da SOBECC. 6a ed. São Paulo: SOBECC; 2013.

18. Galhardo Junior C. Sala cirúrgica híbrida: uma nova realidade no Brasil e no mundo. Rev Bras Cardiol [Internet]. 2013 [cited on 2017 Mar 2];26(1):8-10. Available from: http://pesquisa.bvsalud.org/brasil/ resource/pt/lil-679823

19. Lynn Bell. Using OR patient Classification for Staffing Assignments. AORN J [Internet]. 2015 [cited on 2017 May 23];101(6):639-49. Available from: http://www.aornjournal.org/article/S0001-2092(15)00234-3/pdf

20. Jo KI, Shin JW. Can maximum surgical blood order schedule be used as a predictor of successful completion of bloodless surgery? ANN Lab Med [Internet]. 2013 Mar [cited on 2017 May 02];33(2):11620. Available from: https://www.ncbi.nlm.nih.gov/pmc/articles/ PMC3589636 